\title{
A Distribuição Espacial da Produção Científica e Tecnológica Brasileira: uma Descrição de Estatísticas de Produção Local de Patentes e Artigos Científicos*
}

\author{
Eduardo da M otta e Albuquerque \\ CEDEPLAR/UFM G \\ Rodrigo Simões \\ CEDEPLAR/UFM G e PROPPG/PUC M inas \\ Adriano Baessa \\ CEDEPLAR/UFM G \\ Bernardo Campolina \\ $\mathrm{PBH}$ \\ Leandro Silva \\ CEDEPLAR/UFM G
}

\section{RESUMO}

Tomando como unidade de análise o município, o objetivo deste artigo é descrever a distribuição espacial das atividades científicas etecnológicas no Brasil, a partir de estatísticas de patentes, artigos científicos e pesquisadores.

A hipótese básica équea rel ação produção científica versus produção tecnológica deveser mediada por dimensões da análise econômica que contemplem tanto as variáveis diretamente relacionadas ao sistema de inovação como também seus determinantes espaciais.

Para isto, são real izados alguns exercícios estatísticos, tais como o cál culo de C oeficientes de

\footnotetext{
* Uma versão deste artigo foi apresentada no XXIX Encontro Nacional de Economia - ANPEC (Salvador, dez./2001). Agradecimentos à colaboração dos bolsistas de iniciação científica Ana Luíza Lara, Ana Paula Verona, Fábio Salazar, Regina Fernandes e Túlio Cravo (FACE-UFMG). Wasmália Bivar, Mariana Rebouças (IBGE) e Sinésio Pires Ferreira (MCT) viabilizaram a compatibilização das informações relativas às atividades inovativas no País. Apoio do CNPq e da FAPEMIG são reconhecidos. Os erros são de responsabilidade exclusiva dos autores.
} 
Gini para a produção científica tecnológica; análise de uma M atriz deAssociação G eográficae umaAnálise de Regressão.
Palavras-Chave
Estatística deCiênciaeTecnologia; Distribuição Espacial
CódigosJeL
R00

\section{ABSTRACT}

Thispaper describes thespatial distribution of thescientific and technological activitiesin Brazil, using patents, scientific papers e researchers in a local level data.

Thebasic hypothesisis that therelation between scientific production and technological production has to beunderstood as a mergeamong urban, spatial and innovation variables.

Statistical tests are performed using Gini Coefficients for technological-scientific productions, G eographical Association M atrix and Linear Regression Analysis.

KEYwordS I Scienceand Technology Statistics; Spatial D istribution

JEL-CODES $\mid$ ROO

\section{Introdução}

Tomando como unidade deanálise o município, o objetivo desteartigo é descrever a distribuição espacial dasatividadescientíficas etecnológicasno Brasil, a partir deestatísticas de patentes (uma proxy de capacitação tecnológica), artigos científicos (uma proxy decapacitação científica) epesquisadores (um indicador de recursoshumanosalocados para atividades científicas).

A hipótese básica é que a relação produção científica versus produção tecnológica deveser mediada por dimensões daanáliseeconômica quecontemplem tanto as variáveis diretamenterelacionadasao sistema deinovação como também seus determinantes espaciais.

D uas contribuições podem ser retiradas das informações aqui apresentadas. Por um lado, para os pesquisadores das atividadesinovativasno País, a apresentação desua distribuição espacial podeexplicitar características estruturais do sistema de inovação brasileiro, tais como a sua concentração esua desigual daderegional. Para ospesquisadores deeconomia regional a disponibilidadedeinformações estatísticas 
sobre a distribuição de atividades inovativas pode acrescentar um elemento importante para a aval iação da dinâmica espacial. Com efeito, o papel da dimensão científico-tecnológica na determinação da distribuição espacial das atividades econômicas tem crescido ao longo do tempo e vem sendo tratado de forma continuadana literatura.

0 artigo está organizado em seis seções. N a primeira érealizada a revisão da literatura. $\mathrm{N}$ a segunda, as principaiscaracterísticas do sistema deinovação brasileiro são apresentadas. N a terceira, os dados são descritos. N a quarta, são comparadas as distribuições das atividades produtivas einovativas. $\mathrm{N}$ a quinta, são realizados os testes estatísticos para a avaliação da articulação entre o espaço urbano e a dimensão científica etecnológica. A sexta parte conclui o trabalho.

\section{Revisão da literatura}

0 papel da interação entre as dimensões científica e tecnológica é amplamentediscutido naliteratura sobresistemas nacionais deinovação.

N elson \& Rosenberg (1993) apontam o entrelaçamento entre essas duas dimensões, destacando-o como uma das características principais dos sistemas deinovação. N elson \& Rosenberg (1993:6) resumem as complexas interações entre essas duas dimensões destacan do que a ciência tanto "lidera como segue" ("science asa leader and a follower") o progresso tecnológico.

O utras evidências desseduplo papel podem ser encontradasna literatura.

Em primeiro lugar, Rosenberg (1982:141-159) discute "quão exógena é a ciência". N esse trabal ho, R osen berg ressal ta como os fatores econômicos determinam, até certo ponto, o progresso da ciência, explicitando como 0 progresso tecnológico antecede e estimula o progresso científico. Tal papel pode ser identificado de diversas formas, destacando-se que a tecnologia é uma fonte de questões e problemas para a ciência; e que a tecnologia é um "enorme depósito de conhecimento empírico para ser investigado eavaliado pelos cientistas" (Rosenberg 1982:144).

Em segundo lugar, K levorick et al. (1995) investigam no sentido oposto esta mesma relação, ao apresentar evidências empíricas sobre o papel das universidades e da ciência como fonte de oportunidades tecnológicas para a 
inovação industrial. Esse estudo mostra como os diferentes setores industriais avaliam aimportância relativa das universidades eda ciência paraa sua capacitação inovativa. Essa avaliação explica porque as firmas gastam recursos próprios para monitorar e acompanhar a evolução da pesquisa acadêmica. Especialmente em áreas dealtatecnologia, identificam-sefluxos deconhecimento intensos correndo dainfra-estrutura científica para os setoresindustriais.

Em terceiro lugar, N arin et al. (1997) encontram evidências empíricas do vínculo crescenteentrea ciência (fundamentalmentefinanciadapelo setor público) ea capacidadeinovativa daindústria dosEstados U nidos.

Para os objetivos destetrabalho, esses estudos demonstram a relevância das duas dimensões da atividadeinovativa, enfatizando a divisão detrabal ho entreas mesmas e identifican do a interação e osfeedbacksmútuos entre a infra-estrutura científica e a produção tecnológica nos países desenvolvidos. D esta forma, essa literatura sugere que, para a sustentação do crescimento econômico contemporâneo, tais interações devem estar operando deforma sistêmica.

Também o papel da proximidade geográfica para a articulação entre esses dois componentes do sistema de inovação tem sido discutido pela literatura sobre sistemas locais/regionais de inovação e pela literatura sobre os "spillovers" de conhecimento. Essa segundalinha, em particular, investiga se para a captura do conhecido e, amplamente constatado, efeito de "spillover" (Griliches, 1992) é necessária a proximidade espacial. Jaffe (1989), e Acs et al. (1992) ressaltam a importância da proximidadegeográfica, para a economia dosEstadosU nidos, na relação entreuniversidadesefirmas.

Por sua vez, Audretsch \& Feldman (1996:639), recolhem evidências que indicam que "... indústrias nas quais os spillovers de conhecimento são mais prevalentes - isto é, cujo $P \& D$ industrial, a pesquisa universitária e o trabalho especializado são mais importantes - têm uma mai or propensão de concentrar espacialmente (to cluster) as atividades inovativas do que indústrias onde as externalidades de conhecimento são menosimportantes".

Ainda do ponto de vista de uma ênfase na proximidade como fator de difusão científica etecnológica, J affeet al. (1993) anal isam a localização de patentes e citações como evidência de geographical knowledge spillovers. M ais que isto, mostram que a dinâmica de citações de patentes e referências segue um padrão maisvinculado à proximidadedo queà concentração relativa deatividadescorrelatas 
depesquisa. Paralelamente, Wallsten (2001), explora as relações deaglomeração e spillovers geográficos da indústria do conhecimento, concluindo também quea proximidadeinfluencia fortemente a difusão das atividades deP\& $D$.

D eum ponto devista eminentemente espacial, vários autores relacionam a vinculação das atividades em $P \& D$ com a presença de equipamentos e serviços urbanos, além da própria densidadeeconômica do espaço como fatores deatração. Camagni (1985), utilizando argumentos marshalianos, discute a dimensão aglomerativa relacionada às atividades de $P \& D$ e sua influência no crescimento dasempresas. M alecki (1984), M arkussen et al. (1985) eAngel (1989) relacionam a densidade econômica urbana com a atração de cientistas e tecnólogos para determinadasáreas. Por suavez, Sivitanidou \& Sivitanides(1995) utilizam variáveis relacionadas às chamadasamenidadesurbanas - segundo G otlieb (1995:1413) "(...) amenitiesmay bedefined asplace-specific goodsor services that enter the utility functions of residents directly" - para o estudo da distribuição espacial intrametropolitana deatividades deP\& D para osEstados Unidos. Frankel (2001) aval ia a local ização defirmas dealta tecnologia concluindo queo "milieu productif" exerceinfluência destacada nesta localização, enfatizando tanto o papel dainfraestrutura como o dosserviçosfinanceiroseda própria densidadeurbana relacionada à aglomeração econômica regional.

\section{Sistema de inovação no Brasil: imaturidade e concentração}

H ávários estudosidentificando o caráter incompleto eimaturo do sistema deinovação brasileiro (Coutinho \& Ferraz, 1994). A imaturidade do sistema pode ser identificada por uma comparação deindicadores de ciência etecnologia com países representativos de outras categorias tecnológicas (Albuquerque, 1999).

U ma das formas de analisar o grau de maturidade do sistema de inovação brasileiro, passa pela avaliação da estruturaindustrial brasileira. U ma desagregação da estrutura industrial brasileira de acordo com níveis tecnológicos (segundo proposta da OCDE, 1996), realizada pelo IBGE, indicou que apenas $8,1 \%$ da receita líquida da indústria brasileira provêm de setores classificados como dealta tecnologia. Segundo esseestudo, "na estrutura industrial brasileira predominam os setores de baixa emédia baixa tecnologias (62\%)" (IBGE, 2000:20). 
I dentificada a imaturidade do sistema de inovação brasileiro, énecessário apresentar algumas características distintivas do País. 0 caráter continental e federativo do País exige uma análise das diferenças regionais, ao mesmo tempo que é uma introdução para o papel potencial da construção de sistemas locaise estaduais deinovação no País.

Além do atraso relativo, o Brasil apresenta duas outras particularidades importantes: sua dimensão continental e as disparidades e diferenças regionais existentes. Essas particularidades sugerem a importância de políticas para 0 desenvolvimento local. Cassiolato \& Lastres(1999) apresentam um efforço inicial para captar as diferenças estaduaismais importantesem termos de especializaçõese características dosarranjos produtivoseinovativos dos principais estados brasileiros.

O s dados aqui apresentados, baseados em estatísticas de artigos, patentese pesquisadores, contribuem paraa caracterização mais precisadas diferenças regionais.

Em primeiro lugar, aTabelal apresenta um quadro geral das disparidades estaduais, destacando-sea diferença entre osindicadores de renda edeatividades científica etecnológica (patentes, artigoscientíficose pesquisadores). N a mesma

TABELA I

Média da participação no PIB a custo de fatores, participação no total de patentes, participação no total de artigos científicos e participação relativa dos pesquisadores por Unidade da Federação (1988-1996)

\begin{tabular}{lcrccc}
\hline UF & Participação no PIB & $\begin{array}{r}\text { Distribuição das } \\
\text { patentes - INPI }\end{array}$ & $\begin{array}{c}\text { Distribuição das } \\
\text { patentes - USPTO* }\end{array}$ & $\begin{array}{c}\text { Distribuição dos } \\
\text { Artigos }\end{array}$ & $\begin{array}{c}\text { Participação Relativa } \\
\text { dos pesquisadores }\end{array}$ \\
\hline SP & 0,37 & 0,5368 & 0,5224 & 0,4688 & 0,3238 \\
RJ & 0,11 & 0,1073 & 0,2006 & 0,1737 & 0,1488 \\
MG & 0,10 & 0,0737 & 0,0353 & 0,0685 & 0,0969 \\
RS & 0,07 & 0,0934 & 0,0802 & 0,0616 & 0,0880 \\
PR & 0,06 & 0,0586 & 0,0267 & 0,0368 & 0,0639 \\
PE & 0,02 & 0,0094 & 0,0038 & 0,0307 & 0,0467 \\
DF & 0,02 & 0,0155 & 0,0048 & 0,0260 & 0,0273 \\
SC & 0,03 & 0,0403 & 0,0688 & 0,0244 & 0,0314 \\
BA & 0,04 & 0,0126 & 0,0067 & 0,0165 & 0,0286 \\
Outros & 0,17 & 0,0524 & 0,0510 & 0,0931 & 0,1445 \\
Total & 1,00 & 1,0000 & 1,0000 & 1,0000 & 1,0000 \\
\hline
\end{tabular}

Fonte: Fundação João Pinheiro, INPI, ISI, CNPq, USPTO - (elaboração própria).

Nota: Devido à co-autoria em alguns artigos a soma dos estados difere do total brasileiro.

* Dados correspondem ao período de 1981 a 2000. 
linha, Barros (2000) apresenta dados sobre desigual dades regionaisna produção técnico-científica e quadros que demonstram o peso de São Paulo no sistema deinovação brasileiro.

Analisando a Tabela I, podemos enfatizar que cinco estados da região Sul-Sudeste respondem por 70\% do PIB, 84\% da produção tecnológica, 79\% da produção científica e $69 \%$ dos pesquisadores.

0 peso deSão Paulo deveser destacado. N o período entre 1988 e 1996, este estado respondeu por $37 \%$ do PIB brasileiro, por $53,7 \%$ das patentes registradasno IN PI epor 46,8\% dosartigoscientíficospublicados. É interessante ressaltar que, segundo dados do CN Pq, a participação relativa de pesquisadores em 2000 émais bem distribuída do quea renda (de acordo com essa fonte, São Paulo tem 32,3\% dos pesquisadores do País).

\section{Descrição dos dados no nível municipal}

Como um dos objetivos deste trabalho é a organização dos dados de atividadescientíficas etecnológicas no nível do município, a partir deestatísticas de pesquisadores (fonte: CN Pq), artigos científicos (fonte: ISI) e patentes e contratos averbados de transferência de tecnologia (fonte: IN PI), é necessário descrevêlos, bem como a metodologia de construção dos bancos de dados.

\subsection{Patentes}

Embora no registro da patente o endereço do titular conste do documento (ver www.inpi.gov.br), oIN PI não tem transferido essesdadospara osseus registros magnéticos. Apenasa informação do(s) estado(s) do(s) titular(es) éapresentada.

Essa informação éimportante para estetrabalho, pois implica a exclusão da análise, das patentes cujos titulares são indivíduos (pessoas físicas). 0 peso dos indivíduos no patenteamento no País é elevado. Em termos de patentes depositadas por residentes no Brasil, entre 1988 e 1996, de um total de 57.640 patentes, 38.802 foram de pessoas físicas e 18.838 de pessoas jurídicas. Em termos de patentes concedidas entre 1980 e1995, deum total de8.309 patentes de residentes, 2.954 foram concedidas a pessoas físicas. Essa participação 
importante se mantém junto ao USPT 0, onde entre 1981 e 2000 as pessoas físicas responderam por 33\% das patentes, em um total de 870 concedidas.

O s dados apresentados, portanto, restringem-se a patentes cujos titulares são pessoas jurídicas. A identificação do município foi realizada através do cotejamento dosdadosfornecidospelo IN PI (envolvendo aidentificação do titular) com as informações da RAIS (que articula o titular com um município). Do cruzamento entre as informações do IN PI com a RAIS é construído o banco de dados queé descrito nesta seção.

O sdados do IN PI apresentam informaçõessobre 7.040 diferentesempresas, titulares de 23.919 patentes e/ou contratos de transferência de tecnologia, que entre 1990 e 2000 registraram patentese/ ou averbaram contratos detransferência de tecnologia. 0 cruzamento dessas informações com a RAIS possibilitou a identificação dalocalização municipal de 4.201 empresas, representando 17.587 patentes. Possi velmente, como o banco das patentes refere-sea um período mais amplo (1988 a 1999) e a RAIS a um único ano (1997), algumas firmas que depositaram patentes entre o final da década dos anos 1980 eo início da década dosanos 1990 podem ter desaparecido (por falência, fusão, aquisição, etc.). Também é de se destacar o peso de pequenas firmas no total das patentes. D o total das firmas presentes nos dados do IN PI, 4.001 (56,83\% do total) depositaram apenas uma patente.

O número demunicípios com pelo menosum titular local deuma patente é 512. O s dez municípios com maior produção tecnológica respondem por 53,69\% das patentes identificadas. A Tabela II descreve os dados gerais da distribuição depatentes.

Fonte: INPI (2000), RAIS (1999). (elaboração própria). 
O M apa 1 apresenta a distribuição das patentes por município no País. É importante destacar que apenas quatro municípios (São Paulo, Rio de) aneiro, Campinas eJ oinville) detêm mais do que 500 patentes.

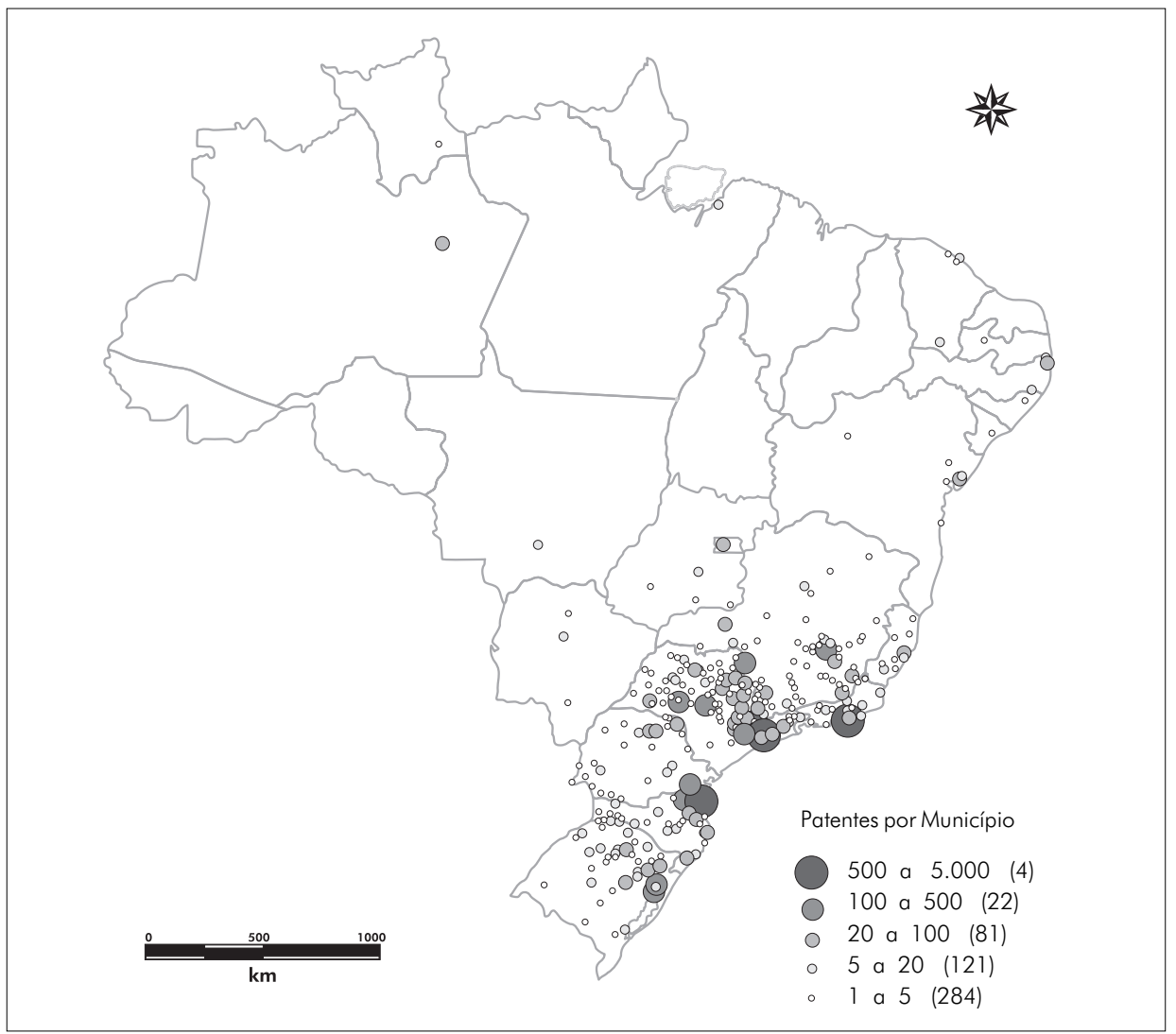

Fonte: IBGE, Malha digital do Brasil (1994).

\subsection{Artigos científicos}

O s dados sobreartigos científicos baseiam-se em informações coletadas no site www.webofscience.fapesp.br, fornecidos pelo Institute for Scientific Information (ISI). U tilizando-se como critério de seleção o Science C itation Index (que exclui revistas da área de humanas e de artes), os 9.668 artigos 
publicados com autores filiados a instituições localizadas no Brasil (em 1999) foram gravados a partir de consultaà Internet. A partir desta coleta, um banco de dados foi construído de forma a tornar operacional izáveis as informações relevantes (autores e suas instituições, nome da revista). '

A restrição a apenas 1999 é uma limitação importante da análise. M as, dada a dificuldade da tarefa de montagem do banco ea escassez de informações com essenível de detalhe, os dados apresentados a seguir devem contribuir para esse mapeamento preliminar dos recursos científicos disponíveis no País.

0 número de municípios com pelo menos um autor participante de uma instituição local é226. O s dez municípios com maior produção científica respondem por $69 \%$ da produção científica nacional. A Tabela III descreve os dados gerais da distribuição de artigos.

O M apa 2 apresenta a distribuição dos artigos por município. É importante destacar que apenas dois municípios (São Paulo e Rio de Janeiro)

\footnotetext{
1 Um artigo pode ter sido elaborado por diversos co-autores. Esses co-autores podem ser pesquisadores de instituições diferentes ou de diferentes departamentos de uma mesma instituição (ou uma combinação entre instituições diferentes e departamentos diferentes). Assim sendo, as informações do ISI podem citar para cada artigo diversas referências de endereço. Para o caso de Minas Gerais, por exemplo, em 1999, este participou com 1.122 artigos e, nas tabelas que fazem uma análise regional do estado, apresenta 2.332 referências de endereços. Ou seja, quase duas referências de endereço, em média, para cada artigo. Isso é um indicador claro de cooperação científica no interior do estado (não é possível, entretanto, afirmar se essa é a proporção de instituições por artigo, pois essas diferentes referências podem representar departamentos de uma mesma instituição). Em termos do total nacional, foram encontrados 9.678 artigos que contêm 32.559 referências de endereços.
} 
ultrapassam a marca de 3.000 artigos, e apenas outros quatro (C ampinas, São Carlos, Belo H orizonte e Porto A legre) ultrapassam a marca de 1.000 artigos.

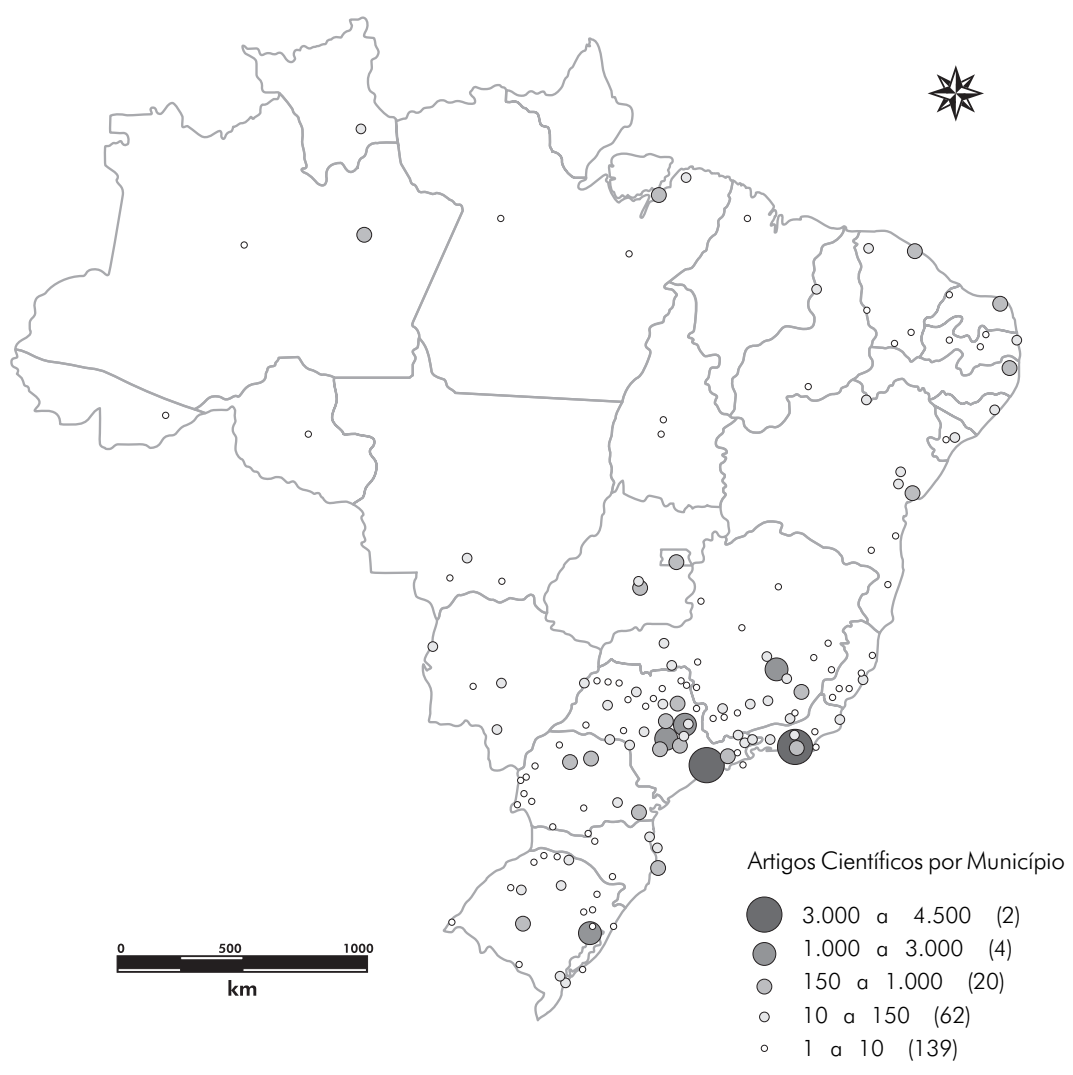

Fonte: IBGE, Malha digital do Brasil (1994). INPI (2000)

Esses dados permitem calcular a "especial ização científica" dos estados. ${ }^{2}$ A Tabela IV apresenta as duas maiores especial izações de cada estado do País (eseus respectivosíndices de especialização).

2 "índice de especialização científica" é calculado por analogia ao Quociente Locacional (QL). Fonte: Haddad et al., 1999. É resultado do seguinte cálculo: $Q \operatorname{lij}=\left[\left(A_{\mathrm{ij}} / \mathrm{A}_{\mathrm{it}}\right) /\left(\mathrm{A}_{\mathrm{tit}} / \mathrm{A}_{\mathrm{tt}}\right)\right]$; onde $\mathrm{A}$ significa produção de artigos, o índice i as áreas do conhecimento, o índice j a região associada e o índice t o total do País. 


\section{TABELA IV}

Relação dos "índices de especialização científica" maiores que a unidade, de acordo com as grandes áreas de conhecimento (CNPq) (1999)

\begin{tabular}{|c|c|c|}
\hline UF & Grande Área CNPq & QL \\
\hline$A C$ & Ciências Agrárias & 8,09 \\
\hline $\mathrm{AL}$ & Ciências Exatas e da Terra & 1,70 \\
\hline \multirow[t]{2}{*}{ AM } & Ciências Biológicas & 2,08 \\
\hline & Ciências Agrárias & 1,66 \\
\hline \multirow[t]{2}{*}{ BA } & Ciências da Saúde & 1,22 \\
\hline & Ciências Biológicas & 1,13 \\
\hline CE & Ciências Agrárias & 2,31 \\
\hline \multirow[t]{2}{*}{ DF } & Ciências Biológicas & 1,44 \\
\hline & Ciências Agrárias & 1,35 \\
\hline \multirow[t]{2}{*}{ ES } & Engenharias & 1,72 \\
\hline & Ciências Exatas e da Terra & 1,21 \\
\hline \multirow[t]{2}{*}{ GO } & Ciências Agrárias & 1,93 \\
\hline & Ciências Biológicas & 1,10 \\
\hline \multirow[t]{2}{*}{ MA } & Ciências Agrárias & 2,54 \\
\hline & Ciências Exatas e da Terra & 1,17 \\
\hline \multirow[t]{2}{*}{ MG } & Ciências Agrárias & 2,94 \\
\hline & Ciências Biológicas & 1,05 \\
\hline MS & Ciências Agrárias & 4,74 \\
\hline \multirow[t]{2}{*}{ MT } & Ciências Biológicas & 1,79 \\
\hline & Ciências Agrárias & 1,44 \\
\hline PA & Ciências Biológicas & 1,64 \\
\hline \multirow[t]{2}{*}{ PB } & Engenharias & 1,77 \\
\hline & Ciências Exatas e da Terra & 1,33 \\
\hline \multirow[t]{2}{*}{$P E$} & Ciências Agrárias & 1,38 \\
\hline & Engenharias & 1,19 \\
\hline $\mathrm{PI}$ & Ciências Agrárias & 2,16 \\
\hline \multirow[t]{2}{*}{$P R$} & Ciências Biológicas & 1,43 \\
\hline & Ciências Agrárias & 1,38 \\
\hline \multirow[t]{2}{*}{ RJ } & Engenharias & 1,19 \\
\hline & Ciências Exatas e da Terra & 1,17 \\
\hline \multirow[t]{2}{*}{ RN } & Engenharias & 1,51 \\
\hline & Ciências Exatas e da Terra & 1,49 \\
\hline \multirow[t]{2}{*}{$\mathrm{RO}$} & Ciências Agrárias & 3,60 \\
\hline & Ciências da Saúde & 2,55 \\
\hline \multirow[t]{2}{*}{$R R$} & Ciências Agrárias & 1,35 \\
\hline & Ciências Exatas e da Terra & 1,07 \\
\hline \multirow[t]{2}{*}{ RS } & Ciências da Saúde & 1,34 \\
\hline & Ciências Agrárias & 1,19 \\
\hline \multirow[t]{2}{*}{ SC } & Engenharias & 1,69 \\
\hline & Ciências Biológicas & 1,03 \\
\hline SE & Ciências Agrárias & 3,43 \\
\hline \multirow[t]{2}{*}{$S P$} & Ciências da Saúde & 1,22 \\
\hline & Engenharias & 1,07 \\
\hline TO & Ciências Biológicas & 4,12 \\
\hline
\end{tabular}

Fonte: ISI (2000); ULRICH'S (1987); CNPq, (2000) - (elaboração própria). 


\subsection{Pesquisadores}

Esta seção baseia-se em dados do CN Pq, disponibilizados através do programaPROSSIGA. Estebanco de dadosapresentaum conjunto de instituições e de grupos de pesquisa, identificando as áreas de atuação dos grupos e o total de pesquisadores envolvidos. É um banco de dados em construção, que tem sido aprimorado em termos de cobertura e de qualidade de informações nos últimos anos (C N Pq, 2000: A pesqui sa no Brasil). Em sua versão do ano 2000, o diretório indica a existência de 48.781 pesquisadores, distribuídos em 224 instituições investigadas.

0 número demunicípios com pelo menos um pesquisador identificado em uma instituição local é 156. O s dez municípios com maior número de pesquisadores envolvem $53,57 \%$ do total nacional. A Tabela $V$ descreve os dados gerais da distribuição de pesquisadores por municípios. 


\section{Comparando a distribuição das atividades produtivas e inovativas $^{3}$}

\section{Procurando seguir sugestão de Suzigan (2000), cal culamos edescrevemos os C oeficientes de G ini para atividades produtivas, tecnológicas e científicas. ${ }^{4}$ A TabelaVI descreveosíndicesencontradosparatodososestadosbrasileiros.}

TABELA VI

Coeficientes de Gini por Unidade da Federação para patentes e contratos averbados no INPI (1990-2000), artigos registrados no ISI (1999), pesquisadores no CNPq (2000) e quantidade de trabalhadores RAIS (1999) preliminar

\begin{tabular}{lccrc}
\hline UF & Patentes & Arrigos & Pesquisadores & Emprego \\
\hline SP & 0,9627 & 0,9917 & 0,9895 & 0,8714 \\
ES & 0,9642 & 0,9927 & 0,9985 & 0,7961 \\
RS & 0,9667 & 0,9965 & 0,9919 & 0,8354 \\
SC & 0,9711 & 0,9966 & 0,9882 & 0,8062 \\
PR & 0,9799 & 0,9952 & 0,9927 & 0,8279 \\
RJ & 0,9872 & 0,9937 & 0,9917 & 0,8854 \\
MG & 0,9883 & 0,9967 & 0,9953 & 0,8546 \\
GO & 0,9900 & 0,9991 & 1,0000 & 0,8868 \\
SE & 0,9910 & 0,9949 & 0,9878 & 0,8514 \\
MS & 0,9912 & 0,9853 & 0,9805 & 0,7790 \\
PE & 0,9915 & 0,9994 & 0,9990 & 0,8525 \\
CE & 0,9921 & 0,9991 & 0,9999 & 0,8592 \\
AL & 0,9925 & 0,9997 & 0,9969 & 0,8329 \\
BA & 0,9937 & 0,9982 & 0,9960 & 0,8805 \\
PB & 0,9964 & 0,9958 & 0,9933 & 0,8695 \\
PA & 0,9980 & 0,9989 & 1,0000 & 0,8931 \\
DF & 1,0000 & 0,9559 & 1,0000 & 0,9963 \\
MT & 1,0000 & 0,9968 & 1,0000 & 0,8024 \\
AM & 1,0000 & 0,9993 & 1,0000 & 0,9745 \\
MA & 1,0000 & 0,9997 & 1,0000 & 0,9174 \\
PI & 1,0000 & 0,9997 & 1,0000 & 0,9249 \\
RR & - & 1,0000 & 0,900 & 0,9922 \\
TO & - & 0,9942 & 0,9858 & 0,9015 \\
RN & - & 0,9996 & 0,9973 & 0,8807 \\
AP & - & 0,9686 & 1,0000 & 0,9478 \\
RO & - & 1,0000 & 1,0000 & 0,8583 \\
AC & - & 0,9958 & 0,9000 & 0,9503 \\
Brasil & 0,9873 & & 0,9937 & 0,8903 \\
\hline
\end{tabular}

Fonte: INPI (2000); ISI (2000); CNPq (2000); RAIS preliminar (1999) - (elaboração própria).

3 Esta seção detém-se na avaliação dos índices de concentração. Dada a limitação de espaço, outro tipo importante de comparação não pode ser aqui realizada: a identificação de possíveis articulações entre especializações econômicas, científicas e tecnológicas dos estados. Trabalho sobre Minas Gerais (Albuquerque, 2001) identificou uma articulação entre as especializações econômicas e industriais (indústria extrativa mineral, indústrias metalúrgica, siderúrgica e agropecuária), as especializações científicas (grandes áreas - ciências agrárias, biologia-e pequenas áreas - zootecnia, agronomia, engenharia metalúrgica e de materiais, farmacologia e biologia geral) e tecnológicas (metalurgia básica e extração de minerais). Silva e Simões (2002), avançam nesta direção procurando identificar, em nível microrregional, a relação entre oportunidades tecnológicas e desenvolvimento científico no Brasil.

4 Para se calcular o índice de Gini, primeiro estratifica-se a amostra organizando-a decrescentemente por estrato (ex.: 500 até $\infty, 200$ até $499, \ldots, 0$ até 10), depois encontra-se a proporção das quantidades de valores na amostra que atendem a cada estrato, assim como a proporção da soma desses valores. Acumula-se essas proporções para as quantidades (X) e para as somas (y), sendo o último estrato igual à unidade. Então aplica-se a fórmula Gini $=\left(\sum x_{i} \cdot y_{i+1}\right)-\left(\sum x_{i+1} \cdot y_{i}\right)$, onde $\mathrm{i}$ é o estrato atual e itl é o estrato seguinte. 
O s valores para o Brasil foram os seguintes:

1) emprego: 0,8903;

2) pesquisadores: 0,9873;

3) produção científica: 0,9958;

4) produção tecnológica: 0,9937.

É importantenotar quea concentração das atividades produtivas, embora elevada, é inferior à concentração encontrada para os tópicos científicos e tecnológicos. Este resultado é contrário ao encontrado para o caso norteamericano, onde o $\mathrm{G}$ ini médio de valor adicionado interindustrial é 0,56 e 0 Gini de inovação é 0,30 (Audretsch \& Feldman, 1996:635).

Para o Brasil, no caso específico da região Sudeste, a partir dos dados da Tabela VI (acima) e dos M apas 3 e 4 (respectivamente para patentes e artigos científicos), é possível verificar a complementaridade existente nas regiões metropolitanas, destacadamente a deSão Paulo (RM SP).

MAPA 3

Região Sudeste, Brasil: Patentes por Município (1990-2000)

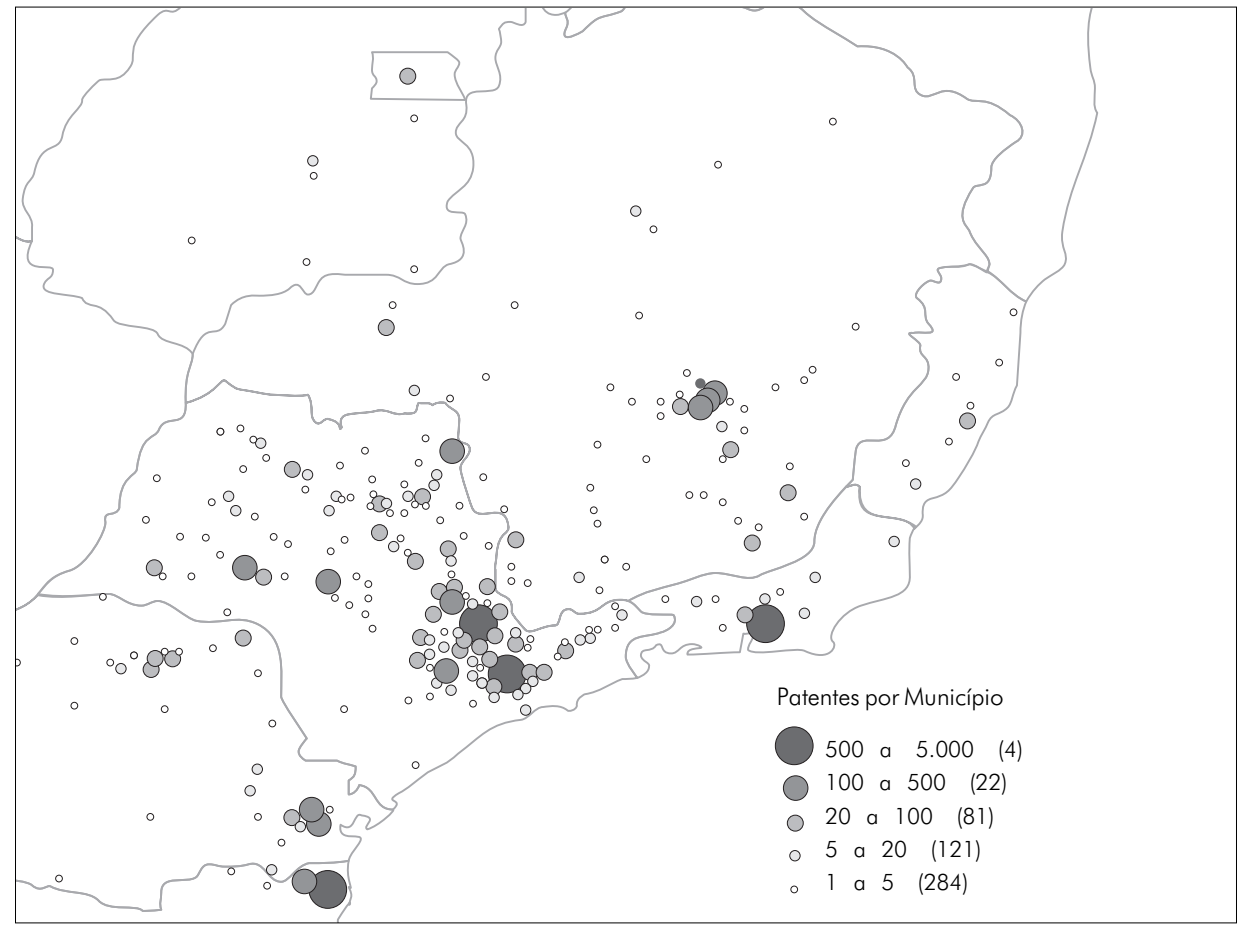

Fonte: IBGE, Malha digital do Brasil (1994).

INPI (2000). 
Região Sudeste: Artigos Científicos por Município (1999)

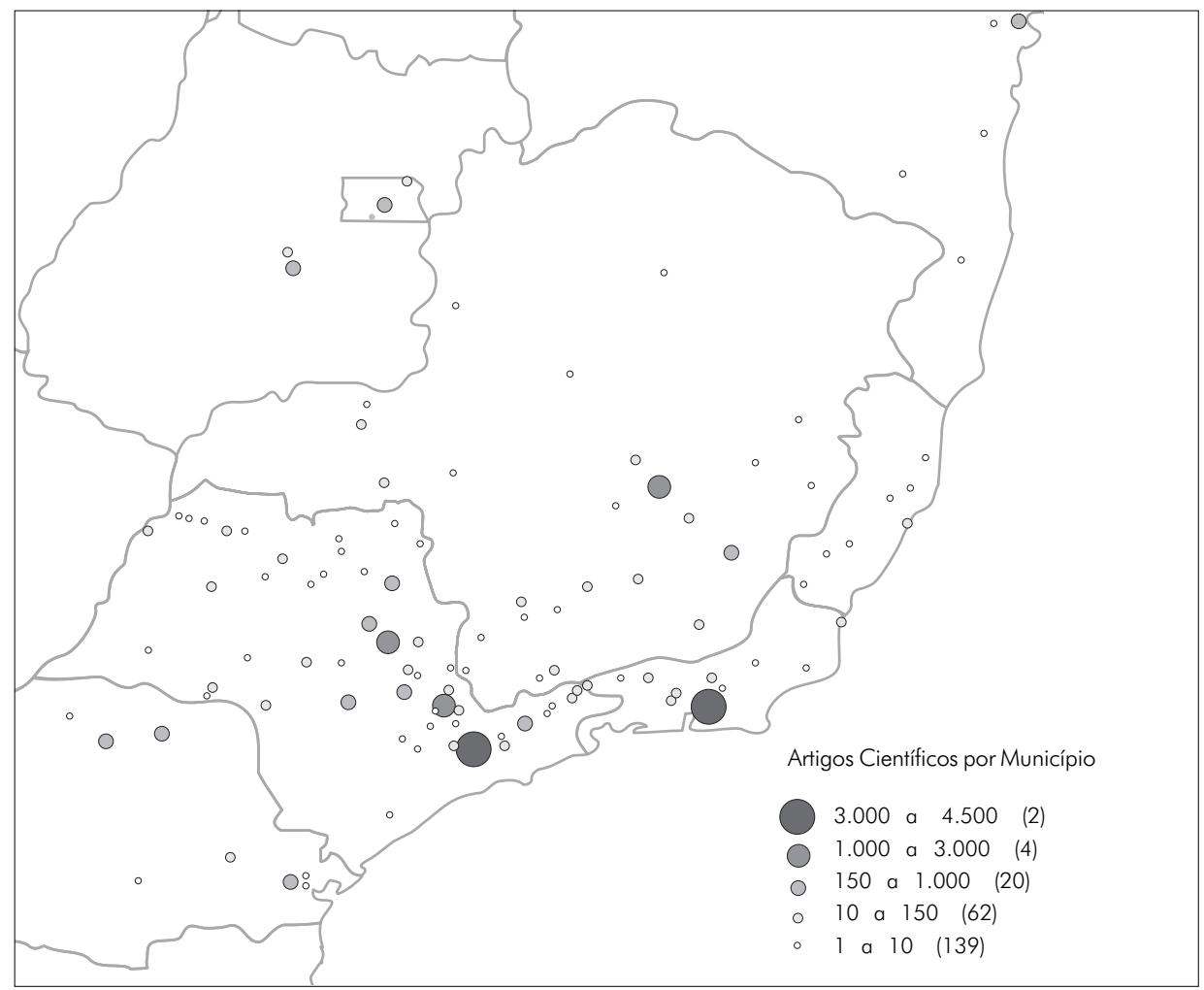

Fonte: IBGE, Malha digital do Brasil (1994).

ISI (2000).

N os M apas 3 e 4 a predominância do município-sede em relação ao Brasil fica clara tanto para a produção tecnológica como para a científica. M ais que isto, podemos notar a nítida interdependência existente entreo município de São Paulo e os demais municípios da RM SP. Se São Paulo possui elevados valores para as duas variáveis - por sua importância e destaque econômico, urbano ecientífico no País - os municípios de seu hinterland imediato possuem uma relação artigos/patentes muito baixa, indicando aquilo que destacamos anteriormentena parte 3 do trabalho. N otável também éa elevada participação dosmunicípios do macroeixo C ampinas-São Carlos-Ribeirão Preto na produção tanto deartigos científicos como de patentes. Esta relativa complementaridade na RM SP pode ser também introdutoriamente anal isada se compararmos os Coeficientes de Gini de São Paulo em relação aos demais estados, tanto da 
região Sudeste como de todo o Brasil (Tabela VI). O s valores do Gini para artigose patentes, apesar deelevadíssimos- 0 quemostra a brutal concentração científico- tecnológica brasileira como destacado anteriormente- são osmenores do País, sendo o de "patentes" devalor inferior, o quepode indicar a importância do processo de desconcentração produtiva do estado a partir de 1970.

$\mathrm{N}$ a Região M etropolitana do Rio de aneiro (RM RJ) esta dinâmica éum pouco menos evidenciada faceà relativa fragilidade do entorno econômico do município-sede. Se este apresenta el evados valores tanto para patentes como para artigos científicos, apenas N iterói (UFF) e em menor escala Seropédica (U FRRJ) aparecem com al guma relevância na produção científica eD uque de Caxias (REDUC) na produção de patentes.

A Região M etropolitana de Belo H orizonte (RM BH ), por fim, apresentase numa hierarquia inferior às anteriores. D o ponto de vista dos artigos é 0 único município com produção destacada na região. D o ponto de vista das patentes al guns outros municípios- Contagem, Betim eSanta Luzia, tal como enfatizado anteriormente - apresentam resultados certamente vinculados à dimensão espacial da dinâmica metropolitana.

\section{Articulando o urbano com a dimensão científico-tecnológica}

D ada a condição periférica do Brasil eo conseqüenteestágio incompleto de construção do seu sistema nacional de inovação, é razoável esperar que a distribuição das atividades tecnológicas não seja completamenteexplicada pela distribuição de atividades científicas. Portanto, esta seção apresenta e discute uma "matriz de coeficientes de associação geográfica".

0 ponto de partida para a constituição dessa "matriz de coeficiente de associação geográfica" éuma tabela contendo os 607 municípios que possuem ao menos uma patente ou um artigo ou um pesquisador local. A estas informações devemos agregar uma medida de escal a e densidade econômicourbana, tal como em C amagni (1985) eWallsten (2001) anteriormente citados, a fim de tentar captar a influência espacial pura na determinação da dinâmica científico-tecnológica. Ao pensarmos no conceito christaleriano de "bem superior", associado e determinante da hierarquia urbana, podemos utilizar o 
setor financeiro e bancário como uma proxy de tal densidade. D esta forma tomamoso total deempregados do setores deintermediação financeira (RAIS1999, D ivisões-CN AE 64, 65 e66) como uma medida aproximativa de escala edensidadeeconômica urbana.

É interessante observar que há uma importante desconexão entre esses municípios.

Em primeiro lugar, destacam-semunicípios com patentesesem produção científica: 24 municípios detêm entre 50 e 382 patentes, embora possuam apenas 0 ou 1 artigo publicado (D iadema, $\mathrm{N}$ ovo H amburgo e Betim são exemplos).

Em segundo lugar, municípios com produção científica e sem patentes: 16 municípios têm entre 20 e 195 artigos, embora possuam entre 0 e 1 patente (exemplos são $\mathrm{N}$ atal, Lavras e Campina $\mathrm{G}$ rande).

A explicação dessa desconexão pareceindicar três dimensões:

1) explicação baseada no nível tecnológico do País: coerente com o identificado pela PIA (2000), a predominância de indústriasclassificadas nos setores de baixa tecnologia e média baixa tecnologia sugere que as "externalidades deconhecimento" ainda não são tão relevantes; daí, fatores locacionais "tradicionais" (proximidade de recursos naturais, etc.) serem maisimportantes;

2) explicação baseada em fatores "espaciais": na verdade, trata-se de uma concepção simples, qual seja, de que tanto a oferta de serviços como, neste caso, a distribuição da atividade produtiva no espaço como um todo atende ao conceito christaleriano da Teoria do Lugar Central de "limite crítico", ou seja vai depender da demanda específica pelo setor e da própria existência ou concentração relativa, ou não, de atividades correlatas nas imediações. Este argumento poderia explicar o porquêde certas regiõesintensivas em "desenvolvimento científico", tal como medido aqui, não possuírem a contrapartida tecnológico-produtiva, aqui medidas em patentes. Da mesma forma, a existência de alta concentração de patentes não implica necessariamente, como veremos à frente, a contrapartida em produção científica;

3) explicação baseada no nível de análise: como foi utilizado o nível município, algumas conexões espaciais importantes foram perdidas 
(exemplo: Contagem, B etim e Santa L uzia, por serem a principal base industrial da RMBH, não possuem pesquisadores ou artigos - por ausência virtual de universidades e centros de pesquisa - mas, de acordo com 0 argumento acima, estão localizadas em uma área que permite 0 aproveitamento do conhecimento gerado em Belo H orizonte). C aso o nível de análise fosse microrregião (ou tomasse em conta áreas metropolitanas), a desconexão acima poderia diminuir.

Esses comentários introduzem a discussão da "matriz de coeficientes de associação geográfica", apresentada naTabelaVII.

\begin{tabular}{|c|c|c|c|c|}
\hline Variável & Artigos & Patentes & Pesquisadores & Urbano \\
\hline Artigos & 0 & 0,573413 & 0,240604 & 0,351613 \\
\hline Patentes & 0,573413 & 0 & 0,611358 & 0,369503 \\
\hline Pesquisadores & 0,240604 & 0,611358 & 0 & 0,362333 \\
\hline Urbano & 0,351613 & 0,369503 & 0,362333 & 0 \\
\hline
\end{tabular}

Fonte: ISI (2000); INPI (2000); CNPq (2000); RAIS (1999).

Essa discussão justifica-se por pontos apresentados nas seções 1 e 2 . Especificamente no trabalho de Audretsch \& Feldman (1996), que sugere existir uma maior propensão à articulação espacial (ou proximidadegeográfica) em indústrias de alta intensidade em $P \& D$ (indústria de alta tecnologia, segundo a O CDE, 1996). N a segunda seção, a debilidade do setor de alta tecnologia no Brasil é apontada. Portanto, dado o estágio de desenvolvimento econômico e tecnológico do Brasil, é de se esperar que a articulação entre universidade e indústria ainda seja relativamente mais baixa, o que implica a presença de outros fatores explicativos mais relacionados com as lições da economia regional clássica.

A introdução dedados relativosà densidadeeconômica eao urbano podem contribuir para explicar o padrão existentededistribuição deatividadesinovativas. ${ }^{5}$ Vale dizer, em países de baixo desenvolvimento científico-tecnológico, com

\footnotetext{
5 Mesmo para uma economia como a norte-americana, Audretsch \& Feldman (1996: 635) trabalham com dados relativos à existência de recursos naturais, custos de transporte, concentração da produção e intensidade de capital.
} 
produção científica abaixo do limiar de 150 artigos/milhão de habitantes em 1996 (Bernardes et al., 2001), devemos buscar também fora das vinculações usuais na literatura sobre $C \& T$ a explicação para a relação entre ciência e desenvolvimento econômico. U ma das possibilidadesétentar captar a influência do espaço urbano, pensado como escala e densidade, nesta determinação. Para isto apresentamos aqui duas análises alternativasintrodutórias. A primeira, uma M atriz deC oeficientes de Associação G eográfica(H addad et al., 1989) apresentada naTabela VII . Esta compara as distribuições das variáveis-base de dois blocos de variáveis entre as regiões. Q uanto mais próximo de 0 melhor a associação geográfica, ou seja, o padrão locacional dos setores seaproxima, indicando uma orientação espacial análoga. Formalmente:

$$
C A_{i k}=\frac{\sum_{j}\left(\mid j^{e i}-j^{e k}\right)}{2}
$$

O s elementos desta matriz podem assim ser analisados:

1) a maior correspondência encontrada éentre o total de artigos e o total de pesquisadores $(0,240604)$, retratando uma articulação razoavelmente direta entreum insumo (pesquisadores) eseu produto (artigos científicos); 2) nas correspondências com patentes (segunda linha da Tabela VII), é interessante observar como a dimensão do urbano émais associada do que a de artigos: esse resultado é uma confirmação da sugestão básica deste artigo, indicando como o nível dearticulação do urbano éum importante determinanteno estágio atual de construção do sistema deinovação;

3) a proxy para a dimensão urbana, por sua vez, está com um nível elevado de associação geográfica com a dimensão tecnológica, científica e com a presença de pesquisadores.

Em segundo lugar procuramos descrever um padrão de comportamento das variáveiscientíficas e tecnológicasem relação à variável densidadeeconômicourbana utilizando análise de regressão. Paratanto tomamosas variáveis patentes, artigose densidadeeconômica urbana etestamos várias alternativas, como sesegue. 
A relação entre produção tecnológica, produção científica e escala econômico-urbana éestimada em uma regressão segundo os seguintes modelos: ${ }^{6}$

$$
\begin{aligned}
& \text { Patentes }=\beta_{1}+\beta_{2}(\text { Artigos })^{2}+\beta_{3}(\text { Urbano })^{2}+\varepsilon \\
& \text { Artigos }=\beta_{1}+\beta_{2}(\text { Patentes })+\beta_{3}(\text { Urbano })+\varepsilon
\end{aligned}
$$

A forma quadrática das variáveis explicativas está de acordo com o esperado, no sentido de quequanto maior a produção deconhecimento acumulada (artigos) emaior adensidadeurbana, maior éa probabilidadedestesatuarem sobrea produção tecnológica (patentes) por efeitos de sinergia. Resultados preliminares acusaram, como previsto em D osi, Freeman eFabiani (1994), a presença deheterocedasticidade no modelo. Com efeito, o método GLS o teste de Breuch-Pagan apontou para a rejeição da hipótese dehomocedasticidade (nos resultadosgerados por O LS).

Primeiramenteestimamosduas regressões com os 603 municípios brasileiros que apresentam algum valor para patentes ou artigos científicos. 0 M odelo 1, estimado por GLS, relaciona a produção de patentescomo variável dependenteda produção deartigos científicos eda escala econômica-urbana, ambas ao quadrado. Como podemosverificar ambasas variáveis independentes são significativas $5 \%$, com sinal positivo e $\mathrm{R}^{2}$ de 0,9342 . Isto mostra claramentequeambasas dimensões - científica eurbana - parecem explicar o comportamento da variável tecnológica no nível municipal. Q uer dizer, quanto maior a escala econômica-urbana equanto maior a produção deartigoscientíficos, maior seráa produção esperada depatentes.

\begin{tabular}{|c|c|c|c|c|}
\hline \multicolumn{5}{|c|}{$\begin{array}{c}\text { QUADRO I } \\
\text { Modelo } 1 \\
\text { (Estimação por GLS) }\end{array}$} \\
\hline \multicolumn{2}{|c|}{$\begin{array}{c}\text { OBS: } 603 \\
F(2,600)=8252,65 \\
\text { Prob }>F=0,0000\end{array}$} & \multicolumn{2}{|c|}{$R^{2}=0,9342$} & \\
\hline Patentes & Coef. & Std. Err. & $t$ & $P>|+|$ \\
\hline Artigos $^{2}$ & 0,0000509 & 0,0000239 & 2,12 & 0,034 \\
\hline Urbano $^{2}$ & 2,31e-07 & $2,52 \mathrm{e}-08$ & 9,15 & 0,000 \\
\hline Cons & 17,23103 & 2,062742 & 8,35 & 0,000 \\
\hline
\end{tabular}
O s resultados desta estimação são apresentados no Q uadro I.

${ }^{6}$ Os resultados foram obtidos utilizando-se o pacote econométrico Intercooled Stata, versão 7. 
N o M odelo 2, utilizando GLS, estimamos uma regressão com a produção de artigos como variável dependente, relacionada também à escala urbana eà produção de patentes. 0 s resultados mostram-se instigantes. N este modelo a variável "patentes" apresenta-se com o sinal invertido ao esperado e, principalmente, não significativa estatisticamente ( $p$-valor $=-0,80)$. I sto parece mostrar que uma hipótese básica do trabalho - explicitada anteriormente parece comprovar-se, ou seja, a dimensão escala econômico-urbana, no caso brasileiro, ainda é mais relevante que a produção de patentes na explicação do comportamento da produção científica. Esse ponto está em linha com trabalho de Rapini (2000), que encontrou no caso do Brasil, na relação entre séries temporais depatentes eartigos, G ranger-causal idadeapenas no sentido deartigos para patentes e não no sentido inverso. N os casos de Coréia do Sul eTaiwan, foram encontradas as $G$ ranger-causalidade nos dois sentidos. A parentemente, em estágios mais avançados de desenvolvimento, as interações recíprocas entre a produção científica e tecnológica sefazem presentes. O Q uadro II mostra os resultados obtido no M odelo 2.

\begin{tabular}{lcccc}
\multicolumn{5}{c}{$\begin{array}{c}\text { QUADRO II } \\
\text { Modelo 2 } \\
\text { (Estimação por } \mathrm{GLS})\end{array}$} \\
\hline & $\begin{array}{c}\text { OBS: 603 } \\
\mathrm{F}(2,600)=34,73 \\
\mathrm{Prob}>\mathrm{F}=0,0000\end{array}$ & \multicolumn{2}{c}{$\mathrm{R}^{2}=0,7989$} & \\
\hline Artigos & Coef. & Est. Err. & $\dagger$ & $\mathrm{P}>|+|$ \\
\hline Patentes & $-0,297736$ & 0,37014 & $-0,80$ & 0,000 \\
Urbano & 0,0474631 & 0,0137104 & 3,46 & 0,000 \\
Cons & 7,467587 & 4,045727 & 1,85 & 0,000 \\
\hline
\end{tabular}

Se reduzimos a nossa amostra para apenas os municípios queapresentam valores diferentes de zero para produção de patentes e produção de artigos científicos ( $n=145$ ) os resultados são semelhantes. N o M odelo 3, estimado por GLS, relacionamos a variável dependente "patentes" com "artigos" e "urbano", obtendo um $\mathrm{R}^{2}$ adj de 0,9534, com coeficientes positivos - tal como esperado ealtamentesignificativos estatisticamente $(p-v a l o r=0)$ (ver Q uadro III). 


\begin{tabular}{|c|c|c|c|c|}
\hline \multicolumn{5}{|c|}{$\begin{array}{c}\text { QUADRO III } \\
\text { Modelo } 3 \\
\text { (Estimação por OLS) }\end{array}$} \\
\hline & $\begin{array}{l}3 S: 145 \\
=1474,45 \\
F=0,0000\end{array}$ & $\begin{array}{r}R \\
\text { Adj }\end{array}$ & & \\
\hline Patentes & Coef. & Est. Err. & $t$ & $P>|t|$ \\
\hline Artigos $^{2}$ & 0,0000474 & $9,61 \mathrm{e}-06$ & 4,93 & 0,000 \\
\hline Urbano $^{2}$ & 2,33e-07 & 1,20e-08 & 19,33 & 0,000 \\
\hline Cons & 38,38299 & 7,526948 & 5,10 & 0,000 \\
\hline
\end{tabular}

O M odelo 4, estimado por GLS, relaciona a produção deartigos (variável dependente) com escala urbana e produção de patentes para os mesmos 145 municípios. O s resultados são análogos ao M odel o 2 com a variável "patentes" tendo o sinal invertido ao esperado e sendo não significativa estatisticamente, com um p-valor=- 0,365 (ver Q uadro IV).

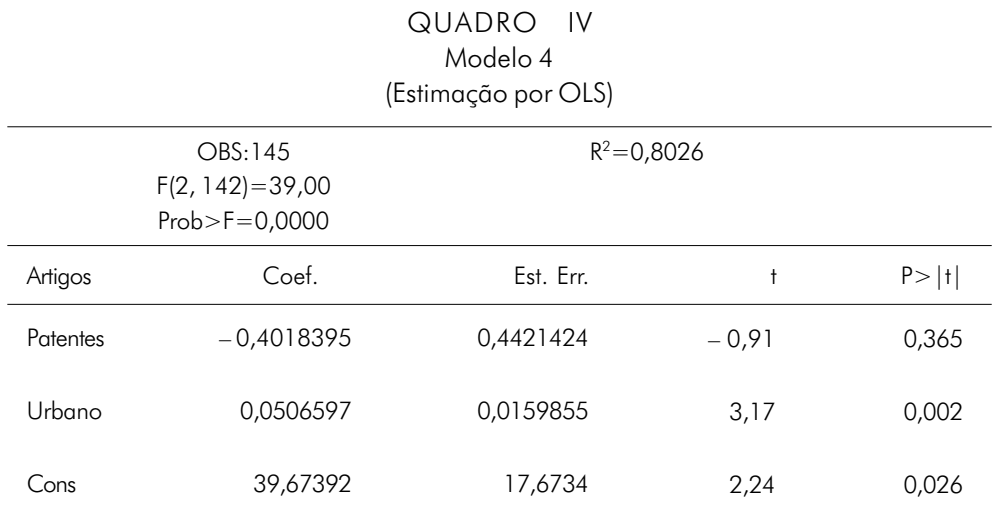

\section{Considerações finais}

As principais conclusões do trabalho são:

1) do ponto de vista da distribuição espacial das atividades inovativas no Brasil, este trabal ho mostrou que ela se encontra altamente concentrada 
no centro-sul do País, notadamente na região Sudeste. Mais que isto foi mostrado que esta concentração, ao contrário do caso americano, é superior à concentração da atividade econômico produtiva;

2) dado o estágio de desenvolvimento do País a explicação da localização das atividades científicas e tecnológicas depende em grande medida de variáveis urbano-espaciais;

3) na comparação entre os estados brasileiros, embora não fuja do padrão geral do País, São Paulo éo queapresenta osmenores coeficientes de concentração de atividades inovativas, além de demonstrar a maior capacidade de complementaridade com seu entorno.

Como a necessária continuidade deste trabalho inicial, três linhas de pesquisa podem ser destacadas:

1) do ponto de vista da avaliação das atividades inovativas, estabelecer correlações entreas especializações econômicas, científicas etecnológicas de cada unidade da federação, efetuando a desagregação setorial das patentes e identificando as disciplinas e subdisciplinas dos artigos científicos;

2) do ponto de vista da análise regional, cabe aprofundar a análise da influência das chamadas amenidades urbanas no processo de localização das atividades de al ta tecnologia;

3) do ponto de vista teórico, trabalhar a articulação desejável e possível entreas dimensões espaciaisetecnológicas em países deindustrialização periférica, como o Brasil. 


\section{Bibliografia}

Albuquerque, E., "N ational Systems of Innovation and N on-O ECD Countries: Notes About a Tentative Typology". Revista de Economia Política, out./dez., 1999.

Sistema Estadual de Inovação em M inas G erais: um balanço introdutório e uma discussão do papel (real e potencial) da FAPEM IG para a sua construção. Belo H orizonte: FAPEM IG (mimeo), 2001.

Amsden, A. H., Asia's N ext Giant: South Korea and Late Industrialization. N ew York/O xford: O xford University, 1989.

Andrade, T. A.; Serra, R. V., "Estimativas para o Produto Interno dos M unicípios Brasileiros: 1975, 1980, 1985 e 1996". Texto para discussão, Nemesis, 2000.

Angel, D. P., "The Labor M arket for Engineers in the U S Semi Conductors Industry". Economic Geography, 65(1), p. 99-112, 1989.

Audretsch, D .; Feldman, M ., "R\& D Spillovers and the Geography of Innovation and Production". American Economic Review, v. 86, n. 3, p. 630-640, 1996.

Barros, F. A. F., "O s D esequilíbrios Regionais da Produção Científica”. São Paulo em Perspectiva, v. 14, n. 3, jul./set., p. 12-19, 2000.

Bernardes, A. T.; Albuquerque, E., Cross over, T hresholds, and Interactions between Science and Technology: a Tentative Model and Initial Notes about Statistics from 120 Countries. Belo H orizonte: CEDEPLAR-UFM G (mimeo), 2001.

Brasil. M inistério do Trabalho, Relatório Anual de Informações Sociais, RAIS, 1997.

Camagni, G., "Spatial Diffusion of Persuasive Process Innovation". Papers of the RSA, 58, p. 83-95, 1985.

Cassiolato, J. E.; Lastres, H. (eds.), G lobalização \& I novação Localizada. Brasília: IBICT, p. 245-278, 1999.

CN Pq, A pesquisa no Brasil (www.cnpq.br), 2000.

Dosi, G.; Freeman, C.; Fabiani, S., "The Process of Economic D evelopment: Introducing Some Stylized Facts and Theories on Technologies, Firms and Institutions". Industrial and Corporate Change, v. 3, n. 1, 1994. 
Fagerberg, J., "Technology and International Differences in Growth Rates". Journal of Economic Literature, v. 32, set., 1994.

Freeman, C.; Soete, L., The Economis of Industrial Innovation. London: Pinter, 1997.

Frenkel, A , "Why Technology Firms Choose to Locate in or N ear M etropolitan Areas". U rban Studies, 38(7), p. 1083-1101, 2001.

Gottlieb, P.D ., "Residential Amenities, Firm Location and Economic D evelopment". U rban Studies, 32(9), p. 1413-1436, 1995.

"Amenities as an Economic D evelopment Tool: Is There Enough Evidence?" Economic D evelopment Q uartely, 8(3), p. 270-285, 1994.

H addad, P. R.; Boisier, S.; Ferreira, C. M . C.; Andrade, T. A., Economia Regional:

Teorias e M étodos de Análise. Fortaleza, BN B/ETEN E, 1989.

IBGE, Pesquisa Industrial, v. 17, Empresa - 1998. Rio de Janeiro: IBGE, 2000. Institute of Scientific Information, (webofscience.fapesp.br), 2000.

Jaffe, A. B., "Real Effects of Academic Research". American Economic Review, v. 79, n. 5, p. 957-970, 1989.

; Trajtenberg, M .; H enderson, R., "G eographical Localization of Knowledge Spillovers as Evidenced by Patents C itations". Q uaterly Journal of Economics, v. 108, n. 3, p. 577-598, 1993.

Klevorick, A.; Levin, R.; N elson, R.; W inter, S, "On the Sources and Significance of Inter-Industry D ifferences in Technological O pportunities". Research Policy, v. 24, p. 185-205, 1995.

M alecki, E.J., "High Technology and Local Economic Development". Journal of American Planning Association, 50(3), p. 262-269, 1984.

M arkussen, A; $H$ all, P.; Glasmeier, A., High Tech America: theW hat, H ow and Why of Sunrise Industries. Boston, M A: Allen and Irwin, 1986.

N elson, R., Sources of Economic Growth. Cambridge, M ass.: H arvard University, 1996. ; Romer, P., "Science, Economic Growth, and Public Policy". In Smith, B. L.; Barfield, C. E., Technology, R\&D, and the Economy. Washington: The Brookings Institution, 1996.

; Rosenberg, N., "Technical Innovation and National Systems". In N elson, R. (ed.). National Innovation Systems. a Comparative Analysis. N ew York, Oxford: Oxford University, p. 3-21, 1993. 
OCDE, Technology and Industrial Performance. Paris: OECD, 1996.

Q uadros, R.; Brisolla, S.; Furtado, A.; Bernardes, R., "Força e Fragilidade do Sistema de Inovação Paulista", São Paulo em Perspectiva, v. 14, n. 3, jul./set., p. 124-141, 2000.

Rapini, M . S., U ma I nvestigação sobre a Relação de G ranger-causalidade entre Ciência

e Tecnologia para Países em Catching up e para o Brasil. Monografia de Graduação. Belo H orizonte: FACE-UFM G, 2000.

Romer, P., "Endogenous Technological Change". Journal of Political Economy, v. 98, n. 3, 1990.

Rosenberg, N., Inside the Black Box: Technology and Economics. Cambridge: Cambridge U niversity, 1982.

Silva, L.; Simões, R. A., "Infra-Estrutura Científica e as 0 portunidades de D esenvolvimento das M icrorregiões Brasileiras". IV Coloquio Transformaciones Territoriales, M ontevidéu: 2002.

Sivitanidou, R.; Sivitanides, P., "The Intrametropolitan Distribution of $R \& D$ Activities: Theory and Empirical Evidences". Journal of Regional Studies, 35(3), p. 391-415, 1995.

Stern, S.; Proter, M .; Furman, J., "The Determinants of N ational Innovative C apability" (Working Paper 7876). N BER Working Paper Series. Cambridge, M A: N BER, 2000.

Suzigan, W., "Aglomerações Industriais como Foco de Políticas" (Texto da Aula M agna do XXVIII Encontro Nacional de Economia da AN PEC). Campinas: AN PEC, 2000.

U Irich's, International Periodicals Directory, 1987-88. 26ㄹ ed. New York: R. R. Bowker Company, 1987.

USPTO , (www.uspto.gov), 2001.

Wallsten, S.J., "An Empirical Test of Geographical Knowledge Spillovers U sing GIS and Firm-Level Data". Regional Science \& U rban Economics, 31(5), p. 571599, 2001. 